\title{
A Fully Eulerian Formulation for Fluid-Structure-Interaction Problems
}

\author{
Thomas Richter ${ }^{\mathrm{a}, 1}$ \\ ${ }^{a}$ Institute for Applied Mathematics, University of Heidelberg, INF 294, 69120 Heidelberg, \\ Germany
}

\begin{abstract}
In this work, we present a Fully Eulerian framework for fluid-structure interaction (fsi) problems coupling the incompressible Navier-Stokes equations with a hyperelastic solid.

The Fully Eulerian framework is a monolithic implicit variational formulation for the coupled problem. In contrast to the well-established Arbitrary Lagrangian Eulerian (ALE) coordinates, the Fully Eulerian framework formulates both subproblems, fluid and solid, in Eulerian coordinates. This concept circumvents various difficulties connected to ALE coordinates since no artificial domain mapping is used. The formulation is an interface-capturing method and uses an extension of the solid's deformation, the Initial Point Set, to detect the interface location.

By construction, very large deformation as well as topology changes like contact of the solid to the domain boundary or other solid parts are possible.

Keywords: finite elements, fluid-structure interaction, Eulerian, monolithic, interface-capturing

$P A C S$ :
\end{abstract}

\section{Introduction}

We present a monolithic variational finite element method for fluid-structure interaction problems. Emphasis is on applications where very large structural deformations, free movement of the structure within a flow domain and contact of the structure with the domain's boundary, with other structures or selfcontact appears. The formulation presented in this work is Eulerian-Eulerian and a first variant of this novel approach has been suggested by Dunne [11, 12].

There exist countless different approaches to model and simulate fluid-structure interaction problems. Among them, we focus on monolithic models, where the complete problem is formulated in one coupled system including the interface conditions between solid and fluid. Monolithic models allow for implicit solution

Email address: thomas.richter@iwr.uni-heidelberg.de (Thomas Richter) 
schemes, large timesteps and offer the possibility to use sensitivity based error estimation and optimization methods. They are well suited for the simulation of problems with large fluid densities as appearing in hemodynamics [15]. While fluid problems are naturally described in a fixed Eulerian coordinate framework, a Lagrangian, material centered description is the usual basis for solid problems. All monolithic schemes for a fluid-solid interaction must somehow match these two different frameworks.

In Lagrangian or arbitrary Lagrangian methods, the flow problem is mapped onto a matching reference domain. Classical approaches are the ALE method, see $[25,3,31]$ or deforming-spatial-domain / stabilized space-time methods (DSD/SST), see for instance [50, 48]. These formulations have in common, that kinematic and dynamic coupling conditions are easily embedded into trial spaces and established by variational techniques. A drawback of Lagrangian methods is the underlying transformation of the fluid-problem which can break down for large deformations or large solid movements. Lagrangian approaches are interface-tracking methods as the common interface is shared by both subproblems.

The Eulerian-Lagrangian methods use a Eulerian fixed computational mesh for the fluid problem and a Lagrangian mesh for the solid problems. Coupling of the two frameworks is accomplished by using force densities as in the immersed boundary method by Peskin [39] or the immersed interface method [35]. Other approaches introduce additional interface variables and couple the two systems using Lagrange multipliers. Examples are the fictitious domain method [19], or other recent approaches based on the extended finite element method [17]. A survey of interface coupling approaches is given by Felippa et. al. [14]. EulerianLagrangian methods are interface-capturing approaches. The interface is not part of the fixed Eulerian fluid mesh and its location within the computational fluid domain must be captured implicitely as part of the solution scheme. An early approach for capturing the interface is the Volume of Fluid method [26], where a tracking function $\Phi_{V}$ takes the value one in the fluid domain and zero in the solid domain. This function $\Phi_{V}$ is transported with the interface velocity. A proper numerical approximation of $\Phi_{V}$ with a sharp jump from fluid to solid domain is one of the difficulties connected to this scheme. Another possibility for capturing the interface - at first used to model multiphase-flows - is by means of a scalar Level-Set $[38,44]$ function $\Phi_{L}$ which in any point of the domain indicates the signed distance to the interface. The interface itself is given as zero-contour of the Level-Set function. Based on Level-Sets, very efficient and simple numerical schemes exist to describe free-boundary and fluid-structure interactions [34]. Usually, Level-Set representations of the interface lack the ability to reproduce sharp corners. A general problem of Eulerian-Lagrangian methods are the approximation properties close to the interface. Since the fluid elements are cut by the interface and the solution may be discontinuous (or at least not differentiable) across the interface, approximation with standard finite elements is difficult. Here, the extended finite element method helps to improve accuracy $[8,17]$.

Finally, for problems with very large deformation, an Eulerian description of 
the structural problem is desirable [51,37]. Using a fixed Eulerian background mesh, the interface between fluid and solid will freely move in the domain. A Eulerian method will always be of interface-capturing type. Dunne [11, 12] has first implementations of an Eulerian-Eulerian model for the interaction of an incompressible fluid with an hyper-elastic solid. Here, capturing of the interface is accomplished with the Initial Point Set (IPS), a vector-field $\Phi_{\text {IPS }}$ used to transport the complete reference coordinate system. The IPS-method is able to capture interfaces with sharp edges. A finite difference approach for EulerianEulerian fluid-structure interaction based on the Volume of Fluid method [26] is introduced by Sugiyama [47] et. al. Here, the coupling between incompressible fluid and structure is solved with a pressure correction iteration. An Eulerian-Eulerian fluid-structure interaction method based on Level-Set functions is introduced by He \& Qiao [20]. Four Level-Set functions are required to represent the moving structure domain. Two of them take a role similar to the Initial Point Set as introduced by Dunne [11, 12].

The Fully Eulerian formulation introduced in this work is based on extensions of the IPS method [43, 13, 40]. Fluid and solid problem are given in an Eulerian formulation, the interface conditions are embedded in the function spaces and realized by variational load balancing. For capturing the interface the solid's deformation will be extended to only a small layer in the fluid domain. The flow problem is modeled without any transformation and apart from the small interface layer no additional variables must be introduced, making the method very efficient.

In the second section we shortly introduce the required notation. Section 3 is devoted to the governing equations in Eulerian coordinates and deals with the coupling of fluid and solid problem. Details on discretization and the solution scheme are provided in Section 4. Finally, in Section 5 we present different numerical examples demonstrating the scope of this new Fully Eulerian fluidstructure interaction formulation.

\section{Preliminaries}

Let $\Omega \subset \mathbb{R}^{2}$ be a two dimensional domain. At time $t=0$ this domain is split into a non-overlapping partitioning into the fluid part $\hat{\Omega}_{f}:=\Omega_{f}(0)$ and solid part $\hat{\Omega}_{s}:=\Omega_{s}(0)$ with a common interface $\hat{\Gamma}_{i}:=\partial \hat{\Omega}_{f} \cap \partial \hat{\Omega}_{s}$. We will consider problems, where the domain partitioning will change in time, the combined domain $\Omega$ however will be fixed:

$$
\hat{\Omega}_{f} \mapsto \Omega_{f}(t), \quad \hat{\Omega}_{s} \mapsto \Omega_{s}(t), \quad \Gamma_{i}(t)=\partial \Omega_{f}(t) \cap \partial \Omega_{s}(t), \quad \Omega=\Omega_{f}(t) \cup \Omega_{s}(t) .
$$

At time $t=0$ we call $\hat{\Omega}_{f}$ and $\hat{\Omega}_{s}$ the reference configuration. In the context of fluid-structure interaction, $\hat{\Omega}_{s}$ refers to the Lagrangian view-point and $\Omega_{s}(t)$ to the Eulerian. For simplicity, we will consider problems with Dirichlet boundary conditions only and define

$$
\Gamma_{f}(t):=\partial \Omega_{f}(t) \cap \partial \Omega, \quad \Gamma_{s}(t):=\partial \Omega_{s}(t) \cap \partial \Omega .
$$


As function spaces we use the Lebesgue space $L^{2}(\Omega)$ in the domain $\Omega$ or in the (moving) subdomains as well as Sobolev-spaces of $L^{2}$ functions with weak derivatives in $L^{2}$. By $(\cdot, \cdot)_{\Omega}$ we denote the $L^{2}$-inner product and by $\|\cdot\|_{\Omega}$ the usual $L^{2}$-norm on $\Omega$. Further, by $\langle\cdot, \cdot\rangle_{\Gamma}$ we denote the $L^{2}$-inner product on (parts of) the boundary $\Gamma \subset \partial \Omega$, usually the inner interface between the two subdomains.

\section{Eulerian formulation of fluid structure interaction}

In this section we derive the coupled system of equations describing the interaction of an incompressible Stokes or Navier-Stokes fluid with an elastic structure of St. Venant Kirchhoff type. All equations are given in variational formulation. This allows for an easy transformation between Eulerian and Lagrangian coordinate frameworks.

\subsection{Eulerian description of fluid flows}

Fluid problems are naturally given in the Eulerian coordinate framework. Here, the flow domain $\Omega_{f}(t)$ is moving in time along with the interface $\Gamma_{i}(t)$ to the solid domain. On this interface, a no-slip condition is assumed for the viscous fluid. Let $v_{i}(t)$ be an extension of the interface-velocity into the fluid domain. Then, velocity and pressure are given as

$$
\begin{aligned}
v_{f}(t) \in v_{i}(t)+\mathcal{V}_{f}(t), \quad p_{f}(t) \in \mathcal{L}_{f}(t): & \\
\rho_{f}\left(\partial_{t} v_{f}(t)+v_{f}(t) \cdot \nabla v_{f}(t), \phi_{f}\right)_{\Omega_{f}(t)}+\left(\sigma_{f}(t), \nabla \phi_{f}\right)_{\Omega_{f}(t)} & =\rho_{f}\left(f_{f}(t), \phi_{f}\right)_{\Omega_{f}(t)} \\
\left(\operatorname{div} v_{f}(t), \xi_{f}\right)_{\Omega_{f}(t)} & =0 \\
\forall \phi_{f} \in \mathcal{V}_{f}(t), \quad \forall \xi_{f} \in \mathcal{L}_{f}(t) & ,
\end{aligned}
$$

where by $\mathcal{V}_{f}(t)$ and $\mathcal{L}_{f}(t)$ we denote suitable function spaces for velocity and pressure on the moving domain $\Omega_{f}(t)$. The Cauchy-stress tensor is given by

$$
\sigma_{f}:=\rho_{f} \nu_{f}\left(\nabla v_{f}+\nabla v_{f}^{T}\right)-p_{f} I,
$$

where by $\rho_{f}$ we denote the fluid's density and by $\nu_{f}$ its kinematic viscosity.

\subsection{Lagrangian description of a St. Venant Kirchhoff material}

In natural Lagrangian coordinates, elastic structures are modeled by describing the deformation of the reference domain $\hat{\Omega}_{s}:=\Omega_{s}(0)$ via $\hat{T}_{s}(t):=\mathrm{id}+\hat{u}_{s}(t)$ : $\hat{\Omega}_{s} \rightarrow \Omega_{s}(t)$. The computational domain $\hat{\Omega}_{s}$ is fixed. On $\hat{\Gamma}_{i}:=\Gamma_{i}(0)$ we drive the problem by interface-stresses coming from the flow domain. Let $\hat{F}_{s}:=I+\hat{\nabla} \hat{u}_{s}$ be the deformation gradient, $\hat{J}_{s}:=\operatorname{det} \hat{F}_{s}$ its determinant and $\hat{v}_{s}:=d_{t} \hat{u}_{s}$ the solid's velocity. The hyperelastic material is governed by:

$$
\begin{aligned}
\hat{u}_{s}(t) \in \hat{\mathcal{V}}_{s}, \quad \hat{v}_{s}(t) \in \hat{\mathcal{L}}_{s}: & \\
\left(\hat{\rho}_{s} d_{t} \hat{v}_{s}(t), \hat{\phi}_{s}\right)_{\hat{\Omega}_{s}}+\left(\hat{F}_{s}(t) \hat{\Sigma}_{s}(t), \hat{\nabla} \hat{\phi}_{s}\right)_{\hat{\Omega}_{s}} & =\hat{\rho}_{s}\left(\hat{f}_{s}(t), \hat{\phi}_{s}\right)_{\hat{\Omega}_{s}}+\left\langle\hat{g}_{s}(t), \hat{\phi}\right\rangle_{\hat{\Gamma}_{i}} \\
\left(d_{t} \hat{u}_{s}(t), \hat{\psi}_{s}\right)_{\hat{\Omega}_{s}} & =\left(\hat{v}_{s}(t), \hat{\psi}_{s}\right)_{\hat{\Omega}_{s}} \\
\forall \hat{\phi}_{s} & \in \hat{\mathcal{V}}_{s}, \quad \forall \hat{\psi}_{s} \in \hat{\mathcal{L}}_{s} .
\end{aligned}
$$


Again, by $\hat{\mathcal{V}}_{s}$ and $\hat{\mathcal{L}}_{s}$ we denote suitable function spaces on $\hat{\Omega}_{s}$. By $\hat{g}_{s}$ we denote interface-stresses on the interface boundary and by $\hat{\rho}_{s}$ the solid's density (in unloaded reference configuration). With the Lamé coefficient $\mu_{s}$ and shear modulus $\nu_{s}$ the second Piola Kirchhoff stress tensor for a St. Venant Kirchhoff material $[27]$ is given by

$$
\hat{\Sigma}_{s}:=2 \mu_{s} \hat{E}_{s}+\lambda_{s} \operatorname{tr}\left(\hat{E}_{s}\right) I,
$$

where $\hat{E}_{s}:=\frac{1}{2}\left(\hat{F}_{s}^{T} \hat{F}_{s}-I\right)$ is the Green-Lagrange strain tensor.

\subsection{Eulerian description of a St. Venant Kirchhoff material}

The Eulerian version of solid equation (2) is derived by simple integral transformation using the natural mapping to the Eulerian framework $\hat{T}_{s}(t): \hat{\Omega}_{s} \mapsto$ $\Omega_{s}(t)$. First, for an Eulerian coordinate $x:=\hat{x}+\hat{u}_{s}(\hat{x}, t)$ we define on $\Omega_{s}(t)$ the Eulerian deformation $u_{s}(t) \in \mathcal{V}_{s}(t)$ and velocity $v_{s}(t) \in \mathcal{L}_{s}(t)$ :

$$
u_{s}(x, t):=\hat{u}_{s}(\hat{x}, t), \quad v_{s}(x, t):=\hat{v}_{s}(\hat{x}, t) .
$$

These definitions allow us to introduce an inverse mapping $T_{s}(t): \Omega_{s}(t) \rightarrow \hat{\Omega}_{s}$ back to the reference domain, its deformation gradient $F_{s}$ and determinant $J_{s}$ :

$$
T_{s}(x, t):=x-u_{s}(x, t), \quad F_{s}:=\nabla T_{s}=I-\nabla u_{s}, \quad J_{s}:=\operatorname{det} F_{s} .
$$

With the relation $T_{s}(t) \circ \hat{T}_{s}(t)=$ id, it follows in Eulerian coordinates:

$$
F_{s} \hat{F}_{s}=I \quad \Rightarrow \quad F_{s}=\hat{F}_{s}^{-1}, \quad J_{s}:=\hat{J}_{s}^{-1}, \quad E_{s}:=\frac{1}{2}\left(F_{s}^{-T} F_{s}^{-1}-I\right) .
$$

Next, let $\hat{\phi}(t) \in \hat{\mathcal{V}}_{s}$ be arbitrary with Eulerian counterpart $\phi(t) \in \mathcal{V}_{s}(t)$ defined by $\phi(x):=\hat{\phi}(\hat{x})$. Then, it holds:

$$
\hat{\nabla} \hat{\phi}=\nabla \phi F_{s}^{-1}, \quad d_{t} \phi=\partial_{t} \phi+v_{s} \cdot \nabla \phi .
$$

Integral transformation of (2) using (4), (5) and (6) gives the Eulerian formulation of the solid problem:

$$
\begin{aligned}
u_{s}(t) \in \mathcal{V}_{s}(t), \quad v_{s}(t) \in \mathcal{L}_{s}(t): & \\
\left(\hat{\rho}_{s} J_{s}\left(\partial_{t} v_{s}+v_{s} \cdot \nabla v_{s}\right), \phi_{s}\right)_{\Omega_{s}(t)}+\left(\sigma_{s}, \nabla \phi_{s}\right)_{\Omega_{s}(t)} & =\left(J_{s} \hat{\rho}_{s} f_{s}, \phi_{s}\right)_{\Omega_{s}(t)}+\left\langle g_{s}, \phi_{s}\right\rangle_{\Gamma_{i}(t)} \\
\left(J_{s}\left(\partial_{t} u_{s}+v_{s} \cdot \nabla u_{s}\right), \psi_{s}\right)_{\Omega_{s}(t)} & =\left(J_{s} v_{s}, \psi_{s}\right)_{\Omega_{s}(t)} \\
\forall \phi_{s} \in \mathcal{V}_{s}(t), & \forall \psi_{s} \in \mathcal{L}_{s}(t),
\end{aligned}
$$

where the Cauchy stress tensor in Eulerian formulation reads:

$\sigma_{s}=J_{s} F_{s}^{-1} \Sigma_{s} F_{s}^{-T}=J_{s} F_{s}^{-1}\left(2 \mu_{s} E_{s}+\lambda_{s} \operatorname{tr}\left(E_{s}\right)\right) F_{s}^{-T}, \quad E_{s}:=\frac{1}{2}\left(F^{-T} F^{-1}-I\right)$.

In Eulerian framework, the solid's density gets $\rho_{s}(x, t)=J_{s}(x, t) \hat{\rho}_{s}(\hat{x})$. Transition to Eulerian coordinates gives rise to convective terms in the momentum equation as well as the velocity-deformation relation. 


\subsection{Coupled fluid structure interaction problem in Eulerian coordinates}

Coupling of (1) and (7) is accomplished by prescribing balancing conditions for velocity and normal stresses on the common interface:

$$
v_{f}(t)=v_{s}(t), \quad \sigma_{f}(t) n(t)=\sigma_{s}(t) n(t) \text { on } \Gamma_{i}(t) .
$$

We realize these conditions by variational techniques. Continuity of velocities is incorporated in one globally defined and "continuous" trial space $\mathcal{V}$ on $\Omega=$ $\Omega_{f}(t) \cup \Omega_{s}(t)$. For $v(t) \in \mathcal{V}$ we use the notation $v_{f}(t):=v_{\mid \Omega_{f}(t)}$ and $v_{s}(t):=$ $v_{\mid \Omega_{s}(t)}$. While looking straightforward, prescribing continuity of the velocities reveals a regularity problem that is discussed in literature [10]. Without further damping, the structure's velocity lacks the required regularity to have a welldefined trace on $\Gamma_{i}(t)$. Although being fundamental, we do not elaborate on this issue here.

The dynamic condition coupling the normal-stresses is realized by means of variationally consistent load evaluation $[7,18]$ choosing common and "continuous" test-functions $\phi \in \mathcal{V}$ with $\phi_{s}:=\phi_{\mid \Omega_{s}(t)}$ and $\phi_{f}:=\phi_{\mid \Omega_{f}(t)}$. Then, the dynamic condition is inherently given $\left(g_{s}=0\right)$ with integration by parts:

$$
\left(\sigma_{f}, \nabla \phi\right)_{\Omega_{f}}+\left(\sigma_{s}, \nabla \phi\right)_{\Omega_{s}}=\left\langle\sigma_{f} n_{f}+\sigma_{s} n_{s}, \phi\right\rangle_{\Gamma_{i}}+\text { "volume integrals", }
$$

with the outward facing normal vectors $n_{f}=-n_{s}$ at the interface $\Gamma_{i}(t)$ with regard to fluid- and solid-domain.

For simplicity of notation we introduce characteristic functions with respect to the two subdomains:

$$
\chi_{f}(x, t):=\left\{\begin{array}{ll}
1 & x \in \Omega_{f}(t) \\
0 & x \in \Omega_{s}(t)
\end{array}, \quad \chi_{s}(x, t):=\left\{\begin{array}{ll}
1 & x \in \Omega_{s}(t) \\
0 & x \in \Omega_{f}(t)
\end{array} .\right.\right.
$$

Combining (1) and (7) the coupled fsi problem is given using the globally defined function spaces:

$$
\begin{aligned}
v \in \mathcal{V}, \quad u_{s} \in \mathcal{V}_{s}, \quad p_{f} \in \mathcal{L}_{f}: & \\
\left(\left(\rho_{f} \chi_{f}+J_{s} \rho_{s} \chi_{s}\right)\left(\partial_{t} v+v \cdot \nabla v\right), \phi\right)+\left(\sigma_{f} \chi_{f}+\sigma_{s} \chi_{s}, \nabla \phi\right) & =\left(\rho_{f} f_{f} \chi_{f}+J_{s} \rho_{s} f_{s} \chi_{s}, \phi\right) \\
\left(\operatorname{div} v_{f}, \xi_{f}\right)_{\Omega_{f}(t)} & =0 \\
\left(J_{s}\left(\partial_{t} u_{s}+v \cdot \nabla u_{s}\right), \psi_{s}\right)_{\Omega_{s}(t)} & =\left(J_{s} v_{s}, \psi_{s}\right)_{\Omega_{s}(t)} \\
\forall \phi \in \mathcal{V}, \quad \xi_{f} \in \mathcal{L}_{f} \quad \psi_{s} \in \mathcal{L}_{s} . &
\end{aligned}
$$

The crux of this very simple looking variational formulation is the partitioning of the domain $\Omega$ into the two subdomains described by (9). The characteristic functions depend on the solution, namely on $u_{s}$, itself. Dunne [12] used the deformation to introduce an additional global vector field, the Initial Point Set $\Phi_{\text {IPS }}$ which at time $t=0$ is the identity $\Phi(x, 0)=x$ and then transported with the solid's velocity $v_{s}$ and its extension to the fluid-domain. This IPS function helps to find a reference location of every Eulerian point. We further 
simplify this approach by directly using the Eulerian inverse transformation $T_{s}(t):=\mathrm{id}-u_{s}(t): \Omega_{s}(t) \rightarrow \hat{\Omega}_{s}$ and its extension to define the characteristic functions:

$$
\chi_{s}(x, t)=\left\{\begin{array}{ll}
1 & x-u(x, t) \in \hat{\Omega}_{s} \\
0 & x-u(x, t) \notin \hat{\Omega}_{s}
\end{array}, \quad \chi_{f}(x, t)=1-\chi_{s}(x, t),\right.
$$

where $u: \Omega \rightarrow \mathbb{R}^{2}$ is an extension of $u_{s}$ to the whole domain. The extension $u$ of the solid's deformation $u_{s}$ establishes the Initial Point Set by the relation $\Phi_{\text {IPS }}(x, t):=x-u_{s}(x, t)$ without the need to introduce additional variables. Note, that for a fluid-coordinate $x \in \Omega_{f}(t)$ we do not necessarily require $x-$ $u_{s}(x, t) \in \hat{\Omega}_{f}$, see $(11)$.

\subsection{Comparison to ALE formulations}

In the classical ALE formulation for FSI problems the structure problem is formulated on the static reference domain $\hat{\Omega}_{s}$ and the flow problem is mapped onto the fixed matching domain $\hat{\Omega}_{f}$ via $\hat{T}_{f}(\hat{x}, t):=\hat{x}+\hat{u}_{f}(\hat{x}, t)$, where $\hat{u}_{f}=$ $\operatorname{ext}_{f}\left(\hat{u}_{s}\right)$ is an extension of the solid's deformation field to the flow domain, see $[31,32,18,1]$. By this transformation, the interface does not move in the computational domain and can easily be resolved with high accuracy. As a drawback, strong nonlinearities are introduced. For comparison with (10), the variational formulation of the coupled ALE system is given by:

$$
\begin{aligned}
&\left(\hat{J} \rho_{f} \partial_{t} \hat{v}_{f}+\rho_{f} \hat{F}^{-1}\left(\hat{v}_{f}-\partial_{t} \hat{T}_{f}\right) \cdot \hat{\nabla} \hat{v}_{f}, \hat{\phi}\right)_{\hat{\Omega}_{f}}+\left(\hat{J} \hat{\sigma}_{f} \hat{F}^{-T}, \hat{\nabla} \hat{\phi}\right)_{\hat{\Omega}_{f}} \\
&+\left(\hat{\rho}_{s} \partial_{t} \hat{v}_{s}, \hat{\phi}\right)_{\hat{\Omega}_{s}}+\left(\hat{F} \hat{\Sigma}_{s}, \hat{\nabla} \hat{\phi}\right)_{\hat{\Omega}_{s}}=\left(\hat{J} \rho_{f} \hat{f}_{f}, \hat{\phi}\right)_{\hat{\Omega}_{f}}+\left(\rho_{s} \hat{f}_{s}, \hat{\phi}\right)_{\hat{\Omega}_{s}} \\
&\left(\operatorname{div}\left(\hat{J} \hat{F}^{-1} \hat{v}_{f}\right), \hat{\xi}\right)_{\hat{\Omega}_{f}}=0, \quad\left(d_{t} \hat{u}_{s}-\hat{v}_{s}, \hat{\psi}\right)_{\hat{\Omega}_{s}}=0,
\end{aligned}
$$

with deformation gradient $\hat{F}:=I+\hat{\nabla} \hat{u}$, its determinant $\hat{J}$ and the stress tensors in Lagrangian formulation:

$$
\hat{\sigma}_{f}:=\rho_{f} \nu_{f}\left(\hat{\nabla} v_{f} \hat{F}^{-1}+\hat{F}^{-T} \hat{\nabla} \hat{v}_{f}^{T}\right)-\hat{p} I, \quad \hat{\Sigma}_{s}:=\mu_{s} \hat{E}_{s}+\lambda_{s} \operatorname{tr}\left(\hat{E}_{s}\right) .
$$

In both formulations, Eulerian and ALE, an extension of the deformation into the fluid domain is introduced. The conceptual difference between the Eulerian and ALE formulation is the use of the deformation-extension to the flow domain. In ALE, with this extension a mapping $\hat{T}_{f}\left(\hat{\Omega}_{f}\right)=\Omega_{f}(t)$ is established used to transform the set of equations. This gives rise to one fundamental problem: if the mapping gets irregular, i.e. $\hat{J}_{f}:=\operatorname{det}\left(\hat{\nabla} \hat{T}_{f}\right) \rightarrow 0 / \infty$, the ALE formulation will break down. This is unavoidable in the case of very large movement (e.g. permanent rotation of a free solid) or topology change (e.g. contact of the solid with the domain's boundary).

In the Fully Eulerian formulation, the extension $u_{f}$ is only used to capture the interface as a look-up function in the definition of $(11)$. Here $T_{f}\left(\Omega_{f}(t)\right) \neq \hat{\Omega}_{f}$ is allowed. We only expect $T_{f}\left(\Omega_{f}(t)\right) \cap \hat{\Omega}_{s}=\emptyset$. This gives more freedom in the choice of the extension's boundary values. Possible regularity problems of 
$u_{f}$ do not influence the overall scheme. For the numerical application it will turn out, that the extension $u_{f}$ is only required in a small layer around $\Gamma_{i}(t)$. Beyond this layer it is sufficient to solve the Navier-Stokes equations without further variables. This leads to a very efficient scheme.

One major drawback of transforming the structure system to Eulerian coordinates is the introduction of convection terms in the equations. Here loss of conservation principles due to numerical diffusion has to be expected. Further, even if the coupled Eulerian system (10) does not include strong nonlinearities at first sight (like it is the case for ALE), implicit domain affiliation leads to nonlinearities in the coupling. This even holds true, if linear models (Stokes and linear elasticity) are coupled. Finally, by having the interface cut through mesh elements, a discretization will require finer meshes to get accuracies similar to those of the ALE approach.

\section{Discretization}

In this section, we describe the discretization scheme used to approximate the fluid-structure interaction system (10). In short, we use a semi-implicit time stepping scheme for temporal discretization and a stabilized equal order finite element discretization in space.

\subsection{Temporal discretization}

Let $0=t_{0}<t_{1}<\cdots<t_{M}=T$ be a subdivision of the time interval $[0, T]$ with (possibly non uniform) step-size $k_{m}:=t_{m}-t_{m-1}$. By $u_{k}=\left(u_{s}^{m}\right)_{m=0}^{M}$, $v_{k}=\left(v^{m}\right)_{m=0}^{M}$ and $p_{k}=\left(p_{f}^{m}\right)_{m=0}^{M}$ we define the solution $U^{m}:=\left\{v^{m}, u_{s}^{m}, p_{f}^{m}\right\}$ at the discrete time-steps $t_{m}$. Every time-step is split into two sub-steps: (1) we march in time $U^{m-1} \rightarrow \tilde{U}^{m}$ using the old domain-partitioning at time $t_{m-1}$. (2) we update the domain partitioning $\Gamma_{i}^{m-1} \rightarrow \Gamma_{i}^{m}$ by extending the new soliddeformation $\tilde{u}_{s}^{m}$ and then project the solution to the new domains $\tilde{U}^{m} \rightarrow U^{m}$.

(1) time-marching. For simplicity, we define $\chi_{f / s}^{m}:=\chi\left(t_{m}\right)_{f / s}, \Omega_{f / s}^{m}:=\Omega_{f / s}\left(t_{m}\right)$, and combine trial-spaces and test-spaces to

$$
U^{m} \in \mathcal{X}^{m}:=\mathcal{V} \times \mathcal{V}_{s}\left(t_{m}\right) \times \mathcal{L}_{f}\left(t_{m}\right), \quad \Phi \in \mathcal{Y}^{m}:=\mathcal{V} \times \mathcal{L}_{s}\left(t_{m}\right) \times \mathcal{L}_{f}\left(t_{m}\right)
$$

For time-stepping, we use the $\theta$-scheme and starting with $U^{m-1} \in \mathcal{X}^{m-1}$ we find the intermediate $\tilde{U}^{m} \in \mathcal{X}^{m-1}$ by

$$
\tilde{U}^{m} \in \mathcal{X}^{m-1}: \quad A^{m-1}\left(\tilde{U}^{m}\right)(\Phi)=0 \quad \forall \Phi \in \mathcal{Y}^{m-1},
$$


with the test-functions $\Phi:=\left\{\phi, \psi_{s}, \xi_{f}\right\}$ and the semilinear form

$$
\begin{aligned}
A^{m-1}\left(\tilde{U}^{m}\right)(\Phi) & =k_{m}^{-1}\left(\left(\rho_{f} \chi_{f}^{m-1}+\tilde{J}_{s}^{m} \rho_{s} \chi_{s}^{m-1}\right)\left(\tilde{v}^{m}-v^{m-1}, \phi\right)_{\Omega}+\left(\operatorname{div} \tilde{v}^{m}, \xi_{f}\right)_{\Omega_{f}^{m-1}}\right. \\
& +\left(\rho_{f}\left(\theta \tilde{v}^{m} \cdot \nabla \tilde{v}^{m}+(1-\theta) v^{m-1} \cdot \nabla v^{m-1}\right), \phi\right)_{\Omega_{f}^{m-1}} \\
& +\left(\rho_{s}\left(\theta \tilde{J}_{s}^{m} \tilde{v}^{m} \cdot \nabla \tilde{v}^{m}+(1-\theta) J_{s}^{m-1} v^{m-1} \cdot \nabla v^{m-1}\right), \phi\right)_{\Omega_{s}^{m-1}} \\
& +\left(\theta \tilde{\sigma}_{f}^{m}+(1-\theta) \sigma_{f}^{m-1}, \phi\right)_{\Omega_{f}^{m-1}}+\left(\theta \tilde{\sigma}_{s}^{m}+(1-\theta) \sigma_{s}^{m-1}, \phi\right)_{\Omega_{s}^{m-1}} \\
& +k_{m}^{-1}\left(\tilde{u}^{m}-u^{m-1}, \psi_{s}\right)_{\Omega_{s}^{m-1}}+\left(\theta \tilde{v}^{m} \cdot \nabla \tilde{u}^{m}+(1-\theta) v^{m-1} \cdot \nabla u^{m-1}, \psi_{s}\right)_{\Omega_{s}^{m-1}} \\
& -\left(\theta \tilde{v}^{m}+(1-\theta) v^{m-1}, \psi_{s}\right)_{\Omega_{s}^{m-1}} \\
& -\rho_{f}\left(\theta f_{f}^{m}+(1-\theta) f_{f}^{m-1}, \phi\right)_{\Omega_{f}^{m-1}}-\rho_{s}\left(\theta \tilde{J}_{s}^{m} f_{s}^{m}+(1-\theta) J_{s}^{m-1} f_{s}^{m-1}, \phi\right)_{\Omega_{s}^{m-1}} .
\end{aligned}
$$

The scalar parameter $\theta \in(0,1]$ controls the time-stepping scheme. For $\theta=1$ we get a variant of the implicit Euler scheme, for $\theta=1 / 2$ the well known CrankNicolson scheme which is of second order in the time. Since we use a first order explicit representation of the domain affiliation by freezing the characteristic functions at the old time step $t_{m-1}$ we cannot expect second order convergence of the overall scheme in the general case. Nevertheless, we choose an implicitely shifted variant of Crank-Nicolson with $\theta=\frac{1}{2}+O(k)$ to avoid excessive numerical dissipation in the fluid equation while formally preserving second order accuracy, see $[23,24,36]$. The intermediate solution $\tilde{U}^{m} \in \mathcal{X}^{m-1}$ at time $t_{m}$ is still given on the old domain partitioning belonging to $t_{m-1}$. Transition to $\mathcal{X}^{m}$ is accomplished in the second substep.

(2) extension step. To generate a new partition $\Gamma_{i}^{m-1} \rightarrow \Gamma_{i}^{m}$ we need to extend the intermediate deformation $\tilde{u}_{s}^{m}$ to the entire domain. Then, given $\tilde{u}^{m}: \Omega \rightarrow$ $\mathbb{R}^{2}$ we can define the new domain partitioning with help of the characteristic functions (11):

$$
\tilde{u}^{m-1}:=\left\{\begin{array}{ll}
\tilde{u}_{s}^{m-1} & \text { in } \Omega_{s}^{m-1} \\
\operatorname{ext}_{f}\left(\tilde{u}_{s}^{m-1}\right) & \text { in } \Omega_{f}^{m-1}
\end{array}, \quad \chi_{s}^{m}:=\left\{\begin{array}{ll}
1 & x-\tilde{u}^{m-1}(x, t) \in \hat{\Omega}_{s} \\
0 & x-\tilde{u}^{m-1}(x, t) \notin \hat{\Omega}_{s}
\end{array} .\right.\right.
$$

Projection of $\tilde{U}^{m-1}$ to the new domain partitioning is accomplished by simply restricting the extensions to the corresponding subdomains:

$$
v^{m}:=\tilde{v}^{m-1}, \quad u_{s}^{m}:=\left.\tilde{u}^{m-1}\right|_{\Omega_{s}^{m}}, \quad p_{f}^{m}:=\left.\tilde{p}^{m-1}\right|_{\Omega_{f}^{m}}
$$

This very simple extension and projection operation will limit the overall accuracy of the scheme to $O(k)$. For obtaining higher order schemes, time-stepping should be performed on space-time slabs similar to the DSD/SST formulation introduced by Tezduyar [49]. For extending the deformation one should use an extension operator which preserves the stiffness of the structure like the biharmonic operator, see [22]. However, since we will introduce a discrete extension on the algebraic level we skip further discussions at this point. 

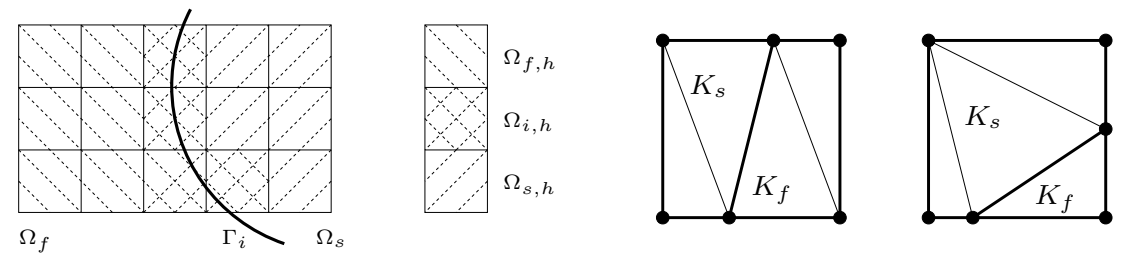

Figure 1: Left: partitioning of a finite element triangulation into overlapping sub-meshes. Right: partitioning of interface-elements into four triangles each for accurate numerical quadrature.

\subsection{Spatial discretization of the time-matching step (1)}

Equation (12) is discretized by a finite element method using continuous, piece-wise polynomial equal order elements. First, by $\Omega_{h}$, we denote a triangulation of the domain $\Omega$ into quadrilaterals. $\Omega_{h}$ consists of open, non-overlapping elements $K \in \Omega_{h}$ and all elements have interior angles which are bound close to $\pi / 2$ in order to prevent too sharp or blunt corners. Details on principals of finite element meshes as well as necessary modifications to deal with adaptive meshes and local mesh refinement are given in the literature $[9,5,2]$. The triangulation $\Omega_{h}$ must not necessarily be aligned with the fluid and structure domain as it is usually the case in ALE formulations. Hence, an element $K$ can be cut by the Interface $\Gamma_{i}(t)$ and be part of both subdomains. In the Fully Eulerian framework the domain partitioning is changing and a matching triangulation would require costly remeshing in every time-step. We define the sub-meshes (see the left sketch in Figure 1):

$$
\Omega_{f / s, h}^{m}:=\left\{K \in \Omega_{h} ; K \cap \Omega_{f / s}^{m} \neq \emptyset\right\}, \quad \Omega_{i, h}^{m}:=\Omega_{f, h}^{m} \cap \Omega_{s, h}^{m} .
$$

By $X_{h}^{m}=V_{h} \times V_{s, h}^{m} \times L_{f, h}^{m}$ we denote a couple of $H^{1}$-conforming isoparametric finite element space of degree $r \geq 1$ which is assembled on the mesh $\Omega_{h}$ and has the usual modification for Dirichlet boundary conditions on $\partial \Omega_{h}$. Since all unknowns (velocity, deformation and pressure) are discretized with order- $r$ finite elements, additional stabilization terms $S_{\mathrm{LPS}}(\cdot)(\cdot)$ are added to the semilinearform $A(\cdot)(\cdot)$ (see (13)). Here, we use the Local Projection Stabilization method to cope with the discrete inf-sup condition. See the survey [4] and [42] for a first application to fluid-structure interaction problems. The stabilized semi-linearform is denoted by $A_{h}(\cdot)(\cdot)$. Further stability problems occur by discretizing the convective terms in the deformation-velocity coupling $\partial_{t} u+$ $v \cdot \nabla u=v$. Here, a stable discretization is accomplished with the consistent streamline diffusion method [33] by modifying the test-space $Y_{h}^{\mathrm{m}, \mathrm{sd}}:=V_{h} \times$ $V_{s, h}^{m, s d} \times L_{f, h}^{m}$.

We emphasize, that deformation $u_{h} \in V_{s, h}$ and pressure $p_{h} \in L_{f, h}$ are not defined on the whole mesh. They appear only on the corresponding sub-meshes. As opposed to ALE formulation, no global extension of the solid deformation is required to solve the fluid-problem. By defining the deformation locally only on 


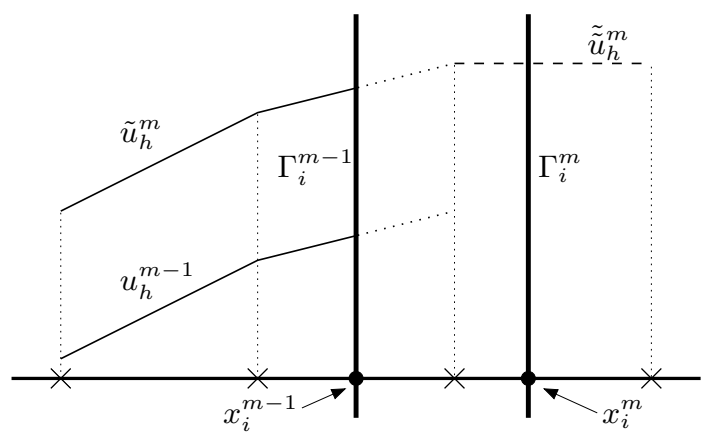

Figure 2: Extension-step (2): old deformation $u_{h}^{m-1}$ and intermediate $\tilde{u}_{h}^{m}$ (on old mesh). Extension $\tilde{\tilde{u}}_{h}^{m}$ into fluid-domain. $x_{i}^{m}$ is new interface location.

$\Omega_{s, h}$ (which is the solid domain plus the small layer of interface elements $\Omega_{i, h}$ ), a significant reduction of numerical effort is reached, as in many applications, the structure fills only a very small part of the entire domain. The stabilized Petrov-Galerkin-formulation of (12) reads:

$$
\tilde{U}_{h}^{m} \in X_{h}^{m-1}: \quad A_{h}^{m-1}\left(\tilde{U}_{h}^{m}\right)\left(\Phi_{h}\right)=0 \quad \forall \Phi_{h} \in Y_{h}^{m-1, \mathrm{sd}},
$$

where $A_{h}(\cdot)(\cdot):=A(\cdot)(\cdot)+S_{\mathrm{LPS}}(\cdot)(\cdot)$.

Since the interface $\Gamma_{i}(t)$ is moving through the domain and crossing mesh elements, one cannot align the mesh nodes with the interface. This is a one severe drawback compared to interface tracking formulations like ALE. Numerical integration thus has to carefully consider the interface regions where the dynamics of the coupled problem are dominated. In Dunne [12] it was proposed to use summed integration formulas to evaluate integrals on elements touched by the interface. For an accurate integration a very large number of integration points is necessary. This method, not taking the specific layout of the interface into account is not efficient. Here, we approximate the interface by a piece-wise linear function and split every element touching the interface into four triangles. In the right sketch in Figure 1 we show examples for the splitting of an element into triangles. Each triangle is integrated with a seven-point Gauss formula [46].

A further approximation problem occurs, since piece-wise polynomial finite element functions $U_{h} \in X_{h}$ are not able to correctly reflect the solution's behavior at the interface. While the velocity is continuous over $\Gamma_{i}$, it's gradient is expected to have a jump. Here, one should use the extended finite element method $[8,17]$ for increasing the interface accuracy.

\subsection{Spatial discretization of the extension-step (2)}

In the extension-step, we first have to extend the intermediate deformation $\tilde{u}_{h, s}^{m}$ into the fluid-domain. We assume, that the time-step $k_{m}$ is small enough to prevent interface-movements larger than one element-size

$$
k_{m}\left\|v_{h}\right\|^{-1} \leq h .
$$


By this assumption it is sufficient to extend the deformation $\tilde{\tilde{u}}_{h}^{m}:=\operatorname{ext}_{h}\left(\tilde{u}_{h, s}^{m}\right)$ to at most one layer of elements into the fluid-domain. Here, we use a simple constant extension. In Figure 2 we give a plot of a one dimensional configuration. After extending the deformation we can locate the new interface-location using the extended $\tilde{\tilde{u}}_{h}^{m}$. The new interface-location $x_{i}^{m}$ belonging to Figure 2 is characterized by the relation

$$
x_{i}^{m}-\tilde{\tilde{u}}_{h}^{m}\left(x_{i}^{m}\right)=x_{i}^{m-1}-u_{h, s}^{m-1}\left(x_{i}^{m-1}\right) .
$$

Since $\tilde{\tilde{u}}_{h}$ is a piece-wise polynomial, this equation is easily solved for $x_{i}^{m}$ with some few steps of a Newton's iteration. Likewise, we extend the intermediate pressure $\tilde{p}_{h, f}^{m}$ one layer into the solid mesh. Given the new partitioning $\Omega_{f, h}^{m}, \Omega_{s, h}^{m}$ we acquire $U_{h}^{m}$ by restriction to the subdomains:

$$
v_{h}^{m}:=\tilde{v}_{h}^{m}, \quad u_{s, h}^{m}:=\left.\tilde{\tilde{u}}_{s, h}^{m}\right|_{\Omega_{s, h}^{m}}, \quad p_{f, h}^{m}:=\left.\tilde{\tilde{p}}_{f, h}^{m}\right|_{\Omega_{f, h}^{m}} .
$$

\subsection{Solution scheme}

In every time-step $t_{m-1} \mapsto t_{m}$ we need to solve a large, nonlinear coupled system of discretized partial differential equations given by (13) and (14):

$$
U_{h} \in X_{h}: \quad A_{h}\left(U_{h}\right)\left(\Phi_{h}\right)=0 \quad \forall \Phi_{h} \in Y_{h},
$$

where we have skipped the superscript " $m$ " denoting the time-step. Given a suitable initial guess $U_{h}^{(0)}$, which is usually the old time-step $U_{h}^{(0)}:=U_{h}^{m-1}$, we approximate the solution by a Newton iteration

$$
W_{h}^{(t)} \in X_{h}: \quad A_{h}^{\prime}\left(U_{h}^{(t)}\right)\left(W_{h}^{(t)}, \Phi_{h}\right)=-A_{h}\left(U_{h}^{(t)}\right)\left(\Phi_{h}\right), \quad U_{h}^{(t+1)}:=U_{h}^{(t)}+W_{h}^{(t)},
$$

where by $A_{h}^{\prime}\left(U_{h}\right)\left(W_{h}, \Phi_{h}\right)$ we denote the Gateaux derivative of $A_{h}(\cdot)(\cdot)$ in direction $W_{h}:=\left\{w_{h}, r_{h}, q_{h}\right\} \in X_{h}$. This derivative is computed analytically based on the variational form (13). The Jacobian of the Navier-Stokes equations is standard and found in the literature. Computing the Jacobian of elastic structure equations, in particular including fluid-structure interactions is more involved, see $[18,1,42]$ for works regarding ALE formulations. Here, due to the separate extension step, the Jacobian does not include derivatives with regard to the movement of the domain. Including these derivatives will be necessary when deriving fully implicit time-stepping schemes which will help to increase stability for larger time-steps. In this case, the derivatives with regard to the domain movement correspond to shape-derivatives as known from topology- and structure-optimization problems [45]. In the context of fluid-structure interactions or free-surface flows, these shape derivatives are analyzed by Brummelen and coworkers [55, 54]. As mentioned, using the semi-implicit time-stepping scheme we only need to evaluate the derivatives with respect to the principal variables which are easily given with help of the following fundamental relations $[27,42]$ :

$$
\frac{\partial F_{s}^{-1}(u)}{\partial u}(\phi)=F_{s}^{-1}(u) \nabla \phi F_{s}^{-1}(u), \quad \frac{\partial J_{s}(u)}{\partial u}(\phi)=J_{s}(u) F_{s}^{-1}(u): \nabla \phi .
$$



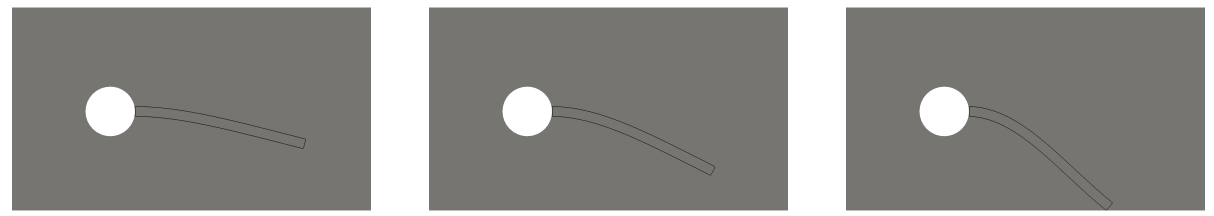

Figure 3: Configuration of the CSM-1 benchmark problem and modifications with larger gravity force. Left $g_{s}=-2$, middle $g_{s}=-4$ and right $g_{s}=-8$.

For completeness, we give the full Jacobian of $A(\cdot)(\cdot)$ for the case of the backward Euler scheme $(\theta=1)$ using the notation $W:=\{w, r, q\}$ and skipping all indices regarding temporal and spatial discretization

$$
\begin{aligned}
A^{\prime}(U)(W, \Phi)= & k_{m}^{-1}\left(\left(\rho_{f} \chi_{f}+J_{s} \rho_{s}\right) w+\rho_{s} J F^{-1}: \nabla r, \phi\right)+\left(\operatorname{div} w, \xi_{f}\right)_{\Omega_{f}} \\
& +\left(\left(\rho_{f} \chi_{f}+\rho_{s} J \chi_{s}\right)(w \cdot \nabla v+v \cdot \nabla w)+\rho_{s}\left(J F^{-1}: \nabla r\right) v \cdot \nabla v, \phi\right) \\
& +\left(\rho_{f} \nu_{f}\left(\nabla w+\nabla w^{T}\right)-q I, \nabla \phi\right)_{\Omega_{f}}+\left(\frac{\partial \sigma_{s}(u)}{\partial u}(r), \nabla \phi\right)_{\Omega_{s}} \\
& +\left(k_{m}^{-1} r+v \cdot \nabla r+w \cdot \nabla u-w, \psi_{s}\right)_{\Omega_{s}}-\left(\rho_{s}\left(J F^{-1}: \nabla r\right), \phi\right)_{\Omega_{s}},
\end{aligned}
$$

with the derivatives of the St. Venant Kirchhoff stress tensor in Eulerian coordinates:

$$
\begin{aligned}
\frac{\partial \sigma_{s}(u)}{\partial u}(r)= & \left(F^{-1}: \nabla r\right) \sigma_{s}(u)+F^{-1} \nabla r \sigma_{s}(u)+\sigma_{s}(u) \nabla r^{T} F^{-T} \\
& +\mu J F^{-1} F^{-T}\left(\nabla r^{T} F^{-T}+F^{-1} \nabla r\right) F^{-1} F^{-T} \\
& +\lambda_{s} J F^{-1} \operatorname{tr}\left(F^{-1} \nabla r F^{-1} F^{-T}\right) F^{-T}
\end{aligned}
$$

A very efficient implementation of these derivatives is possible since most terms, like the product $F^{-1} F^{-T}$ appear very often and must be coded only once. The linear system to be solved in every step of the Newton iteration involves a very large and coupled, ill conditioned matrix given by (15) and (16). In this first work on the Eulerian method we use a direct solver. While multigrid methods are well established for both solid and fluid computations, the application to a coupled fluid-structure interaction problem is very involved. For efficient solving one has to exploit a partitioned structure within the multigrid smoother, see $[21,28,6,16,41]$. Application of a partitioned inner iteration to the Eulerian formulation is difficult, since the interface cuts through mesh elements and no strict partitioning is available.

\section{Numerical Examples}

\subsection{Model validation}

For validation of the Eulerian model we consider a simple fluid-structure interaction benchmark, the CSM-1 problem as proposed by Hron and Turek [29]. 
In this benchmark configuration, we model the deformation of an elastic beam, attached to an obstacle under a gravity force, see Figure 3. In [29] different configurations have been proposed. Here, for validation purposes, we focus on the first CSM-1 test-case. Initially, fluid and solid are at rest, the problem is driven by a gravity force $f_{s}=-J_{s} \hat{\rho}_{s} g_{s} \chi_{s}$ acting on the elastic beam. In the original benchmark configuration [29] $g_{s}=2$ has been used, Wick [52] also published results for $g_{s}=4$ yielding a larger deformation. To exploit the possibilities of very large deformation with the Eulerian approach, we add a further test-case using $g_{s}=8$. We measure the deformation $u_{s}$ in the tip of the beam $A=(0.6,0.2)$ in the stationary limit. In Table 1 we present the deflections in this measurement point on different meshes with decreasing mesh sizes under three different gravity forces. For comparison, we indicate the reference values are stated in $[29,30]$ and $[52,53]$. The complete set of parameters used in this configuration is:

$$
\rho_{f}=\hat{\rho}_{s}=10^{3}, \quad \nu_{f}=10^{-3}, \quad \mu_{s}=5 \cdot 10^{5}, \quad \lambda_{2}=2 \cdot 10^{6}, \quad f=-g_{s} J_{s} \hat{\rho}_{s} \chi_{s}
$$

It is clearly seen, that the Full Eulerian Method yields accurate values which are very close to the reference values cited from the literature. Further, the Eulerian framework is able to increase the gravity force. Considering the testcase $g_{s}=8$, the beam touches the rigid bottom of the flow-chanel, see Figure 3 . Here, no results for comparison are available in the literature.

\begin{tabular}{c|cc|cc|cc} 
& \multicolumn{2}{|c|}{$g_{s}=2$} & \multicolumn{2}{c|}{$g_{s}=4$} & \multicolumn{2}{c}{$g_{s}=8$} \\
mesh size & $u^{x}(A)$ & $u^{y}(A)$ & $u^{x}(A)$ & $u^{y}(A)$ & $u^{x}(A)$ & $u^{y}(A)$ \\
\hline$h_{\min } \approx 0.008$ & 6.372 & 61.84 & 21.22 & 114.54 & 59.846 & 189.74 \\
$h_{\min } \approx 0.004$ & 7.116 & 64.70 & 25.02 & 121.25 & 65.760 & 192.03 \\
$h_{\min } \approx 0.002$ & 7.149 & 66.07 & 25.10 & 122.16 & 66.857 & 192.35 \\
\hline Hron \& Turek [29] & 7.187 & 66.10 & \multicolumn{2}{|c|}{$\mathrm{n} / \mathrm{a}$} & \multicolumn{2}{c}{$\mathrm{n} / \mathrm{a}$} \\
Wick [52, 53] & 7.150 & 64.90 & 25.33 & 122.30 & \multicolumn{2}{c}{ n/a }
\end{tabular}

Table 1: Results for the CSM-1 benchmark problem using increasing volume forces. Functional values on a sequence of meshes. Comparison to reference values taken from the literature using the ALE framework.

\subsection{Contact problem}

Finally, we model the "free fall" of an elastic ball $\Omega_{s}$ with radius $r_{\text {ball }}=0.4$ in a container $\Omega=(-1,1)^{2}$ filled with a viscous fluid $\Omega_{f}$. Figure 4 shows the configuration of this test-case. At time $t=0$, the midpoint of the ball is at $x_{0}=(0,0)$. Since gravity is the only acting force on the solid, the ball will accelerate and fall to the bottom $\Gamma_{\text {bot }}$. At this rigid wall, the ball stops and due to elasticity it will bounce off again. The parameters used for this test-case are given by

$$
\rho_{f}=10^{3}, \quad \hat{\rho}_{s}=10^{3}, \quad \nu_{f}=10^{-2}, \quad \mu_{s}=10^{4}, \quad \lambda_{s}=4 \cdot 10^{4}, \quad f=-J_{s} \hat{\rho}_{s} \chi_{s} .
$$



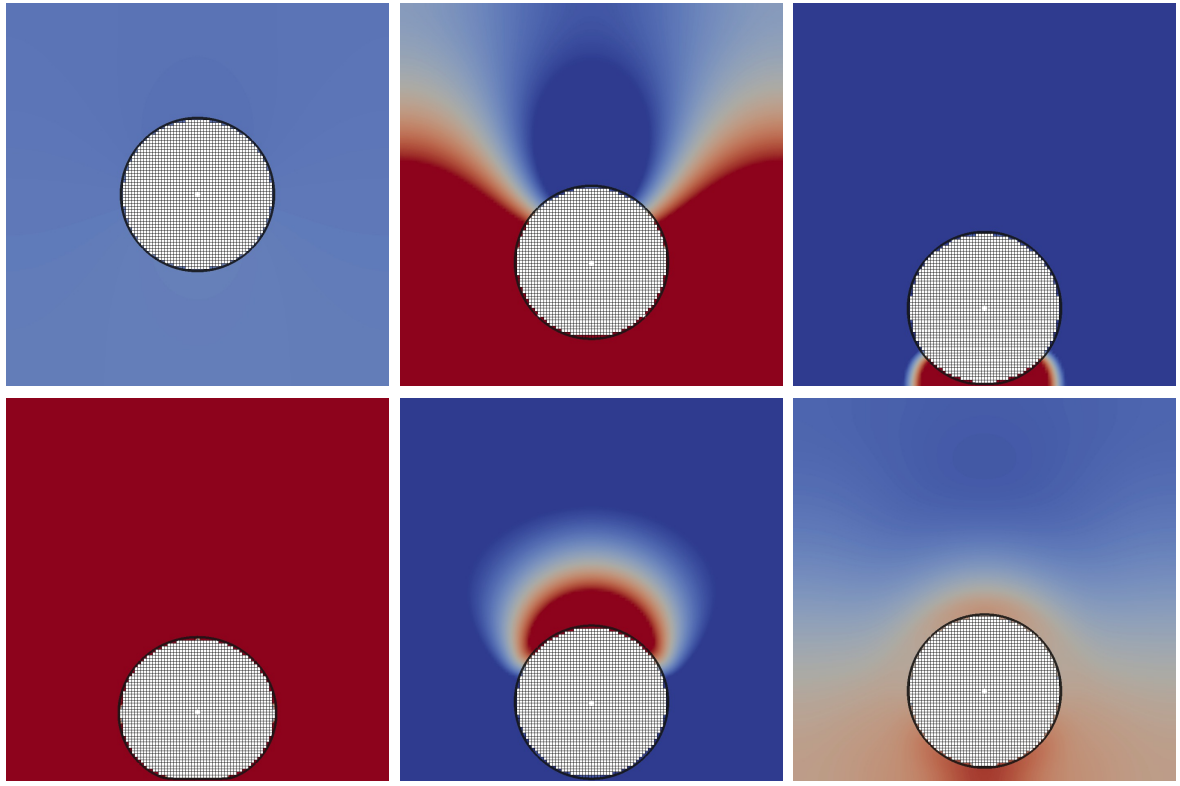

Figure 4: Falling ball bouncing of the bottom wall. Snapshots of the solution at times $t=0$, $t=0.71, t=0.96$ (first contact), $t=1.035$ (biggest deformation), $t=1.125$ (breaking contact) and $t=1.38$ (highest bounce-off).

As functional outputs, we measure the average $y$-displacement and $y$-velocity of the structure as well as the structure's volume:

$$
j_{\mathrm{vol}}(t):=\int_{\Omega_{s}(t)} 1 \mathrm{~d} x, \quad j_{u}(t):=\int_{\Omega_{s}(t)} u_{s}^{y}(t) \mathrm{d} x, \quad j_{v}(t):=\int_{\Omega_{s}(t)} v_{s}^{y}(t) \mathrm{d} x .
$$

\begin{tabular}{c|rrr}
$h$ & 0.0100 & 0.0050 & 0.0025 \\
\hline $2^{-5}$ & -0.4977 & -0.4990 & -0.5006 \\
$2^{-6}$ & -0.5248 & -0.5286 & -0.5298 \\
$2^{-7}$ & -0.5402 & -0.5311 & -0.5315
\end{tabular}

\begin{tabular}{r|rrr}
\multicolumn{1}{l|}{$k$} & 0.0100 & 0.0050 & 0.0025 \\
\hline $2^{-5}$ & 0.320 & 0.348 & 0.365 \\
$2^{-6}$ & 0.318 & 0.369 & 0.396 \\
$2^{-7}$ & 0.357 & 0.388 & 0.404
\end{tabular}

Table 2: Left: maximum (negative) velocity reached in free fall. Right: maximum average velocity after bounce-off. Calculations on three different spatial and temporal meshes.

In Figure 4 we show snapshots of the solution for different times. Figure 5 shows the progress of the functionals as function over time.

In Table 2 we indicate the maximum (negative) velocity that is reached at the time of first contact $t_{C} \approx 0.952$, as well as the maximum velocity that is reached after the first bounce-off $t_{B} \approx 1.105$, see Figure 5 . Computations are 

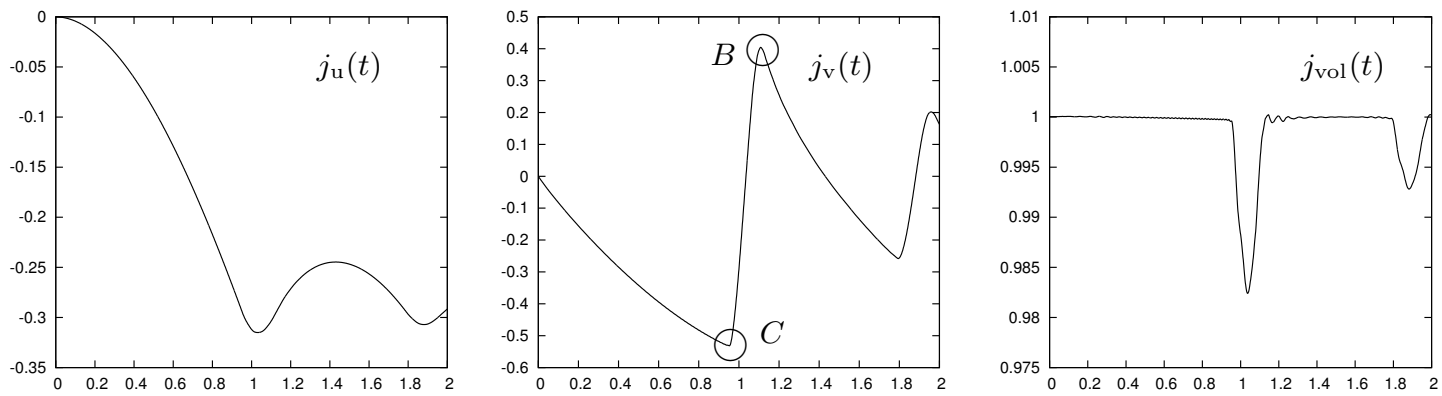

Figure 5: Falling ball: functionals as plot over time. Left: solid's average deformation. Middle: solid's average velocity. Right: solid's relative volume. The two turning points of the velocity for contact (C) and maximum bounce-off (B) are indicated in the middle plot.

done using three different temporal and spatial discretization parameters $h$ and $k$. All meshes are uniform in space and time.

Finally, in Table 3 we give the temporal error with regard to mass conservation

$$
\left\|j_{\text {mass }}(t)-\hat{\rho}_{s} \pi r_{\text {ball }}^{2}\right\|_{L^{2}([0,2])}, \quad j_{\text {mass }}(t):=\int_{\Omega_{s}(t)} J_{s} \hat{\rho}_{s} \mathrm{~d} x .
$$

Here, we observe $O\left(h^{2}\right)$ convergence. Being an interface-capturing method like Level-Sets, this result has to be expected for linear finite elements. The timediscretization parameter $k$ appears to be too small to have a substantial influence on the accuracy.

\begin{tabular}{r|rrr}
\hline \hline$k$ & 0.0100 & 0.0050 & 0.0025 \\
\hline $2^{-5}$ & $2.68 \cdot 10^{-3}$ & $2.66 \cdot 10^{-3}$ & $2.69 \cdot 10^{-3}$ \\
$2^{-6}$ & $7.82 \cdot 10^{-4}$ & $6.95 \cdot 10^{-4}$ & $6.72 \cdot 10^{-4}$ \\
$2^{-7}$ & $2.63 \cdot 10^{-4}$ & $1.92 \cdot 10^{-4}$ & $1.68 \cdot 10^{-4}$ \\
\hline \hline
\end{tabular}

Table 3: Error in mass conservation for the falling ball.

\section{Outlook}

\section{References}

[1] Y. Bazilevs, V.M. Calo, T.J.R Hughes, and Y. Zhang. Isogeometric fluidstructure interaction: theory, algorithms, and computations. Comput Mech, 43:3-37, 2008.

[2] R. Becker and R. Rannacher. An optimal control approach to a posteriori error estimation in finite element methods. In A. Iserles, editor, Acta Numerica 2001, volume 37, pages 1-225. Cambridge University Press, 2001. 
[3] T. Belytschko. Fluid-structure interaction. Comput. Struct., 12:459-469, 1980.

[4] M. Braack and G. Lube. Finite elements with local projection stabilization for incompressible flow problems. Journal of Computational Mathematics, 27:116-147, 2009.

[5] M. Braack and T. Richter. Solutions of 3D Navier-Stokes benchmark problems with adaptive finite elements. Computers and Fluids, 35(4):372-392, May 2006.

[6] E.H. van Brummelen, K.G. van der Zee, and R. de Borst. Space/time multigrid for a fluid-structure-interaction problem. Applied Numerical Mathematics, 58(12):1951-1971, 2008.

[7] G.F. Carey, S.S. Chow, and M.K. Seager. Approximate boundary-flux calculations. Comput. Methods Appl. Mech. Engrg., 50:107-120, 1985.

[8] J. Chessa, P. Smolinski, and T. Belytschko. The extended finite element method (xfem) for solidication problems. Int. J. Numer. Meth. Engrg., 53:1959-1977, 2002.

[9] P.G. Ciarlet. Finite Element Methods for Elliptic Problems. North-Holland, Amsterdam, 1978.

[10] D. Coutand and S. Shkoller. Motion of an elastic solid inside an incompressible viscous fluid. Arch. Ration. Mech. Anal., pages 25-102, 2005.

[11] T. Dunne. An eulerian approach to fluid-structure interaction and goaloriented mesh refinement. Int. J. Numer. Math. Fluids., 51:1017-1039, 2006.

[12] T. Dunne. Adaptive Finite Element Approximation of Fluid-Structure Interaction Based on Eulerian and Arbitrary Lagrangian-Eulerian Variational Formulations. $\mathrm{PhD}$ thesis, University of Heidelberg, 2007. urn:nbn:de:bsz:16-opus-79448.

[13] T. Dunne, R. Rannacher, and T. Richter. Numerical simulation of fluidstructure interaction based on monolithic variational formulations. In G.P. Galdi and R. Rannacher, editors, Comtemporary Challenges in Mathematical Fluid Mechanics. World Scientific, Singapore, 2010.

[14] C.A. Felippa, K.C. Park, and M.R. Ross. A classification of interface treatments for fsi. In H.-J. Bungartz, M. Mehl, and M. Schäfer, editors, Fluid Structure Interaction II, volume 73 of Lecture Notes in Computational Science and Engineering, pages 27-52. Springer, 2010.

[15] G.P. Galdi, R. Rannacher, A.M. Robertson, and S. Turek, editors. Hemodynamical Flows: modeling, analysis and simulation. Birkhäuser Verlag, Basel-Boston-Berlin, 2008. 
[16] M.W. Gee, U. Küttler, and W.A. Wall. Truly monolithic algebraic multigrid for fluid-structure interaction. Int. J. Numer. Meth. Engrg., 85:987-1016, 2010 .

[17] A. Gerstenberger and W.A. Wall. An extended finite element method/lagrange multiplier based approach for fluid-structure interaction. Comput. Methods Appl. Mech. Engrg., 197:1699-1714, 2008.

[18] O. Ghattas and X. Li. A variational finite element method for stationary nonlinear fluid-solid interaction. Journal of Computational Physics, 121:347-356, 1995.

[19] R. Glowinski, T.W. Pan, T.I. Hesla, D.D. Joseph, and J. Périaux. A fictitious domain approach to the direct numerical simulation of incompressible viscous flow past moving rigid bodies: application to particulate flow. $J$. Comp. Phys., 169:363-426, 2001.

[20] P. He and R. Qiao. A full-eulerian solid level set method for simulation of fluidstructure interactions. Microfluidics and Nanofluidics, 11:557-567, 2011.

[21] M. Heil. An efficient solver for the fully coupled solution of largedisplacement fluid-structure interaction problems. Comput. Methods Appl. Mech. Engrg., 193:1-23, 2004.

[22] B.T. Helenbrook. Mesh deformation using the biharmonic operator. Int. J. Numer. Meth. Engrg., pages 1-30, 2001.

[23] J. Heywood and R. Rannacher. Finite element approximation of the nonstationary Navier-Stokes problem. iii. smoothing property and higher order error estimates for spatial discretization. SIAM J. Numer. Anal., 25(3):489$512,1988$.

[24] J. Heywood and R. Rannacher. Finite element approximation of the nonstationary Navier-Stokes problem. iv. error analysis for second-order time discretization. SIAM J. Numer. Anal., 27(3):353-384, 1990.

[25] C.W. Hirt, A.A. Amsden, and J.L. Cook. An arbitrary lagrangian-eulerian computing method for all flow speeds. J. Comp. Phys., 14:227-469, 1974.

[26] C.W. Hirt and B.D. Nichols. Volume of fluid (vof) method for the dynamics fo free boundaries. J. Comp. Phys., 39:201-225, 1981.

[27] G.A. Holzapfel. Nonlinear Solid Mechanics: A Continuum Approach for Engineering. Wiley-Blackwell, 2000.

[28] J. Hron and S. Turek. A monolithic fem/multigrid solver for an ale formulation of fluid-structure interaction with applications in biomechanics. In H.-J. Bungartz and M. Schäfer, editors, Fluid-Structure Interaction: Modeling, Simulation, Optimization, Lecture Notes in Computational Science and Engineering, pages 146-170. Springer, 2006. 
[29] J. Hron and S. Turek. Proposal for numerical benchmarking of fluidstructure interaction between an elastic object and laminar incompressible flow. In H.-J. Bungartz and M. Schäfer, editors, Fluid-Structure Interaction: Modeling, Simulation, Optimization, Lecture Notes in Computational Science and Engineering, pages 371-385. Springer, 2006.

[30] J. Hron, S. Turek, M. Madlik, M. Razzaq, H. Wobker, and J.F. Acker. Numerical simulation and benchmarking of a monolithic multigrid solver for fluid-structure interaction problems with application to hemodynamics. In H.-J. Bungartz and M. Schäfer, editors, Fluid-Structure Interaction II: Modeling, Simulation, Optimization, Lecture Notes in Computational Science and Engineering, pages 197-220. Springer, 2010.

[31] T.J.R. Hughes, W.K. Liu, and T.K. Zimmermann. Lagrangian-eulerian finite element formulations for incompressible viscous flows. Computer Methods in Applied Mechanics and Engineering, 29:329-349, 1981.

[32] A.A. Johnson and T.E. Tezduyar. Mech update strategies in parallel finite element computations of flow problems with moving boundaries and interfaces. Comput. Methods Appl. Mech. Engrg., 119:73-94, 1994.

[33] C. Johnson. Numerical Solution of Partial Differential Equations by the Finite Element Method. Cambridge University Press, Cambridge, UK, 1987.

[34] A. Legay, J. Chessa, and T. Belytschko. An eulerian-lagrangian method for fluid-structure interaction based on level sets. Comput. Methods Appl. Mech. Engrg., 195:2070-2087, 2006.

[35] R. Leveque and Z. Li. The immersed interface method for elliptic equations with discontinuous coefficients and sinuglar sources. SIAM J. Numer. Anal., 31:1019-1044, 1994.

[36] M. Luskin and R. Rannacher. On the smoothing propoerty of the cranknicholson scheme. Applicable Anal., 14:117-135, 1982.

[37] S. Okazawa, K. Kashiyama, and Y. Kaneko. Eulerian formulation using stabilized finite element methods for large deformation solid dynamics. Int. J. Numer. Math. Fluids., 72:1544-1559, 2007.

[38] S. Osher and R. Fedkiw. Level set methods and dynamic implicit surfaces. Applied Mathematical Sciences. Springer, 2003.

[39] C.S. Peskin. The immersed boundary method. Acta Numerica, 11:479-517, 2002.

[40] R. Rannacher and T. Richter. An adaptive finite element method for fluidstructure interaction problems based on a fully eulerian formulation. In H.J. Bungartz, M. Mehl, and M. Sch" afer, editors, Fluid-Structure Interaction II, Modelling, Simulation, Optimization, number 73 in Lecture notes in computational science and engineering, pages 159-192. Springer, 2010. 
[41] T. Richter. A monolithic multigrid solver for 3d fluid-structure interaction problems. submitted to Siam J. Scientific Computing, 2011.

[42] T. Richter. Goal-oriented error estimation for fluid-structure interaction problems. Comput. Methods Appl. Mech. Engrg., submitted 2011.

[43] T. Richter and T. Wick. Finite elements fo fluid-structure interaction in ale and fully eulerian coordinates. Computer Methods in Applied Mechanics and Engineering, 2010. doi: 10.1016/j.cma.2010.04.016).

[44] J.A. Sethian. Level set methods and fast marching methods evolving interfaces in computational geometry. Fluid mechanics, Computer Vi.

[45] J. Sokołowski and J.-P. Zolésio. Introduction to shape optimization, volume 16 of Computational Mathematics. Springer, 1992.

[46] A.H. Stroud. Approximate calculation of multiple integrals. Prentice-Hall, 1971.

[47] K. Sugiyama, S. Li, S. Takeuchi, S. Takagi, and Y. Matsumoto. A full eulerian finite difference approach for solving fluid-structure coupling problems. JCP , 230:596-627, 2011.

[48] K. Takizawa and T.E. Tezduyar. Multiscale space-time fluid-structure interaction techniques. Comput. Mech., 48:247-267, 2011.

[49] T.E. Tezduyar. Stabilized finite element formulations for incompressible flow computations. Adv. Appl. Mech., 28:1-44, 1992.

[50] T.E. Tezduyar and S. Sathe. Modeling of fluid-structure interactions with the space-time finite elements: solution techniques. Int. J. Numer. Math. Fluids., 54:855-900, 2007.

[51] P. van Hoogstraten, P.M.A. Slaats, and F.P.T. Baaijens. A eulerian approach to the finite element modelling of neo-hookean rubber material. Appl. Sci. Res., 48:193-210, 1991.

[52] T. Wick. Fluid-structure interactions using different mesh motion techniques. Computers and Structures, 89:1456-1467, 2011.

[53] T. Wick. Technical report, University of Heidelberg, 2012. Benchmark Results for Fluid-Structure Interaction Problems in ALE Coordinates using Different Mesh Motion Techniques.

[54] K.G. van der Zee, E.H. van Brummelen, I. Akkerman, and R. de Borst. Goal-oriented error estimation and adaptivity for fluid-structure interaction using exact linearized adjoints. Comput. Methods Appl. Mech. Engrg., 200:2738-2757, 2011.

[55] K.G. van der Zee, E.H. van Brummelen, and R. de Borst. Goal-oriented error estimation and adaptivity for free-boundary problems: The shapelinearization approach. SIAM J. on Scientific Computing, 32(2):1093-1118, 2010. 